\title{
Ultra-slow antiproton beam line MUSASHI
}

\author{
Naofumi Kuroda ${ }^{a}$, Hiroyuki A. Torii ${ }^{b}$, Masahiro Shibata ${ }^{a}$, \\ Yugo Nagata ${ }^{b}$, Dániel Barna ${ }^{c}$, Masaki Hori ${ }^{d}$, \\ Akihiro Mohri ${ }^{a}$, Ken-ichiro Komaki ${ }^{b}$, Yasunori Yamazakia, ${ }^{a}$ \\ ${ }^{a}$ RIKEN, Wako, Japan \\ ${ }^{b}$ Institute of Physics, University of Tokyo, Komaba, Tokyo, Japan \\ $c_{\text {KFKI, Budapest, Hungary }}$ \\ $d_{\mathrm{CERN}}$, Genève, Switzerland
}

\begin{abstract}
The ASACUSA collaboration succeeded in producing an ultra-slow antiproton beam source, named MUSASHI, Monoenergetic Ultra-Slow Antiproton Source for High-precision Investigation, consisting of an electro-magnetic trap and low energy antiproton beam transport line. This MUSASHI confined and cooled $1.2 \times 10^{6}$ of antiprotons, and extracted them as ultra-slow antiproton beams with energy of 10-500 eV.
\end{abstract}

\section{Introduction}

The preparation of a large number of antiprotons at extremely low energy is an important step on the road to synthesizing antihydrogen atoms $\left(\bar{p} e^{+}\right)$and antiprotonic atoms, e.g., protonium $(\bar{p} p)$ atom. Such exotic atoms can only be efficiently synthesized from component particles at $\mathrm{eV}$ and lower energies, and this is far below the GeV scale of accelerator-produced antiprotons. Therefore the development of ultra-slow antiproton beams is essential to study the initial process of antiprotonic atom $\left(\bar{p} \mathrm{~A}^{+}\right)$formation [1], to make high precision spectroscopy of $\bar{p} \mathrm{~A}^{+}$, to study collision dynamics in the ionization of atoms by antiprotons [2], and to study nuclear surface structure via antiprotonic atom formation and annihilation $[3,4]$. It is noted that antiprotons provide a unique possibility to study the behavior of "heavy electron" or "negatively charged proton", because other negatively charged heavy particles such as $\pi^{-}$and $\mu^{-}$have finite lifetimes.

The ASACUSA (Atomic Spectroscopy And Collisions Using Slow Antiprotons) prepared ultra-slow antiproton source by the sequential combination of the CERN Antipro- 


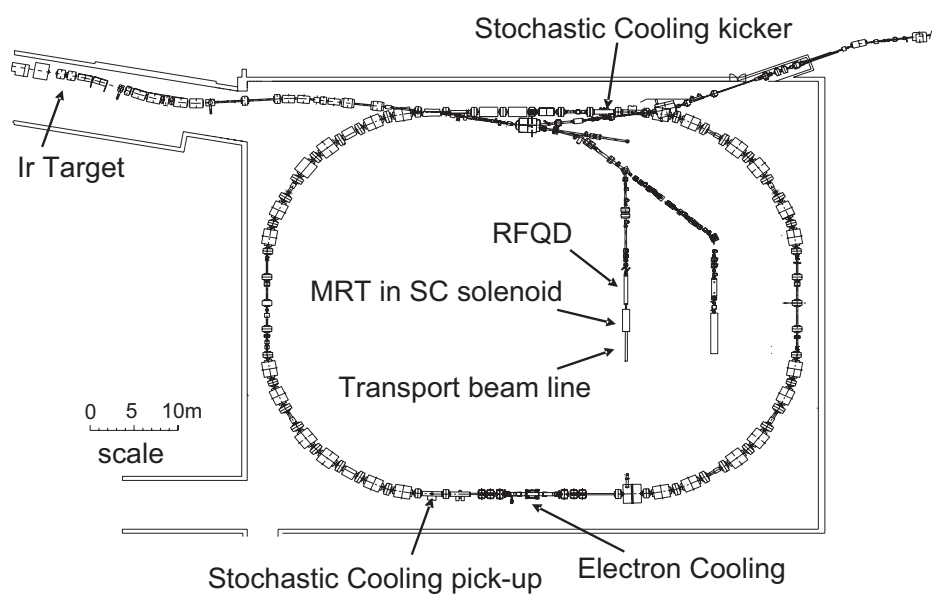

Figure 1: Layout of the Antiprotons Decelerator (AD) at CERN.

ton Decelerator $(\mathrm{AD})$, the radio frequency quadrupole decelerator (RFQD), the multiring electrode trap (MRT), and a low energy antiproton beam transport line.

\subsection{AD and RFQD}

At CERN, the Antiproton Decelerator (AD) decelerates antiprotons with $2.7 \mathrm{GeV}$ kinetic energy to $5.3 \mathrm{MeV}$, and ejects them every $84 \mathrm{~s}$ in $90 \mathrm{~ns}$ long pulses, containing some $2 \times 10^{7}$ particles.

In the conventional experiments where degrader foils were used to reduce antiprotons energy further from the $\mathrm{MeV}$ to $\mathrm{keV}$ scale [5, 6, 7], many incident antiprotons stopped and annihilated inside relatively thick foils, while the rest emerged with too high an energy to be confined effectively in the potential well of any trap. Indeed, the largest number of captured particles so far reported for a typical AD pulse, or shot, was $2.5 \times 10^{4}[8]$. We here replaced this simple degrader foils by the RFQD to suppress antiproton loss in the foil and improve the trapping efficiency. This decelerator, $4 \mathrm{~m}$ in length, reduced the $5.3 \mathrm{MeV}$ AD beam energy between 10 and $120 \mathrm{keV}$. Some $5 \times 10^{6}$ antiprotons within a single shot fulfilled the phase condition required by the acceptance and deceleration by the RFQD, the remainder being transmitted without deceleration $[9,10]$. We expected that the reduced antiproton energy would allow a much larger number of emerging antiprotons to be captured in a trap placed after the RFQD than is normally the case for simple degrader foils.

Since the antiprotons were to spend a long time in the trap, more stringent vacuum conditions were required there to avoid antiproton loss by annihilation on residual gas atoms [7] than in the RFQD, through which they passed only once. Two $90 \mu \mathrm{g} / \mathrm{cm}^{2}$ polyethylenterephtalat (PET) foils located $70 \mathrm{~cm}$ upstream of the MRT were therefore 


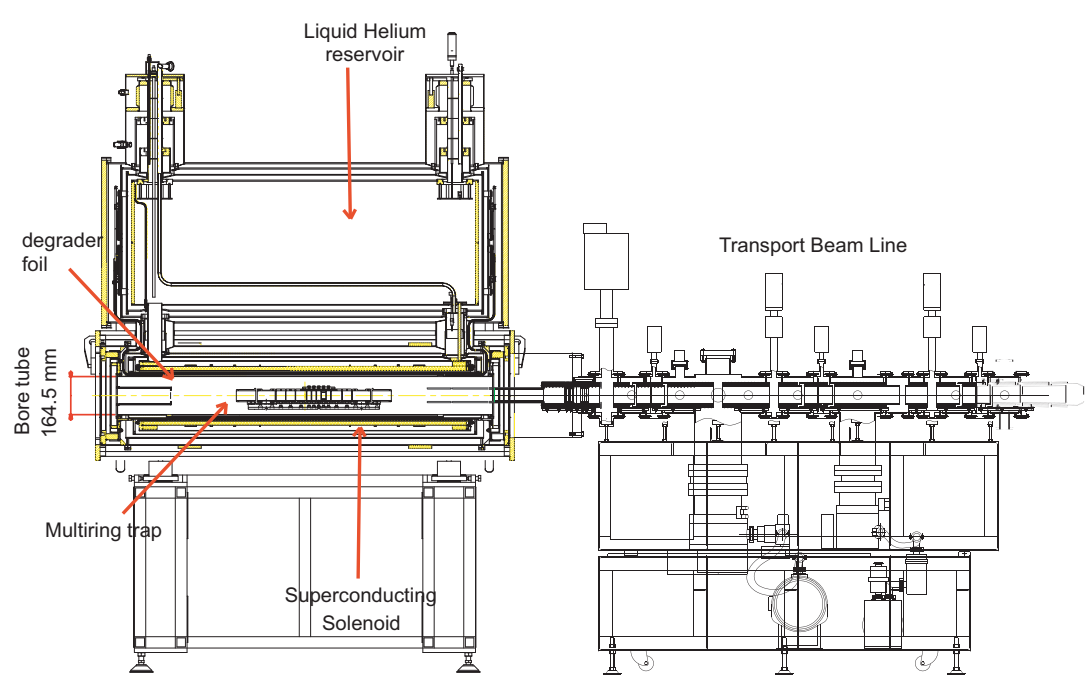

Figure 2: A drawing of MUSASHI.

used to isolate the trap and RFQD vacuum systems, at $10^{-12}$ mbar and $10^{-9}$ mbar, respectively.

Although these were some 800 times thinner than those used at the entrance of the traps described in Refs. [5, 6, 7], antiprotons traversing them unavoidably lost their energy ( $\sim 100 \mathrm{keV})$. The corresponding angular divergence and straggling caused some $30 \%$ of the antiprotons to annihilate on the way through [11], but this is to be compared with corresponding losses in Refs. [5, 6, 7] of $99.9 \%$ in total for the case of stopping direct beam from the $\mathrm{AD}$ at $5.3 \mathrm{MeV}$ by thick degrader foils.

The potential of the entire RFQD could be floated by several tens of kilovolts relative to the ground, and we tuned the value of this floating voltage so as to maximize the capture efficiency of the grounded trap. We set the RFQD beam output energy to be $110 \mathrm{keV}$.

\section{MUSASHI}

MUSASHI (Monoenergetic Ultra-Slow Antiproton Source for High-precision Investigation), composed of two parts, an MRT and a transport beam line for ultra-slow antiproton beams (Fig. 2).

\subsection{Antiproton trap}

The multiring trap (MRT) [12] comprised of 14 ring electrodes lying perpendicular to the beam and on the axis of a superconducting solenoid, as is shown in Fig. 3.

This superconducting solenoid which were made by Oxford Instruments produced $5 \mathrm{~T}$ 